\title{
Densidad estomática de arveja (Pisum sativum) por microscopía electrónica de barrido en relación al rendimiento
}

\author{
Stomatal density of pea (Pisum sativum) by scanning electron microscopy in relation to yield
}

Densidade estomática de ervilha (Pisum sativum) por microscopia eletrônica de varredura em relação ao rendimento

\author{
Reynado Francisco Cherrepano Manrique \\ rcherrepano@unjfsc.edu.pe \\ https://orcid.org/0000-0001-8249-2480
}

Universidad Nacional José Faustino Sánchez Carrión, Perú

\section{Dante Daniel Cruz Nieto}

daniel2262@gmail.com

https://orcid.org/0000-0003-0052-5619

Universidad Nacional José Faustino Sánchez Carrión, Perú

\author{
Yasmin Jesús Vélez Chang \\ yvelez@unjfsc.edu.pe \\ https://orcid.org/0000-0003-0333-8173
}

Universidad Nacional José Faustino Sánchez Carrión, Perú

\author{
José Antonio Legua Cárdenas \\ jlegua@unjfsc.edu.pe
}

https://orcid.org/0000-0002-4978-4980

Universidad Nacional José Faustino Sánchez Carrión, Perú
RESUMEN

El objetivo fue determinar la densidad estomática, por microscopía electrónica, de hojas de arveja cultivada a diferentes dosis de nitrógeno y distanciamientos entre plantas, para obtener mayor rendimiento. Por lo que se instaló el área experimental empleando el Diseño de Bloques Completamente al Azar, con seis tratamientos que constaron de $\mathrm{F} 1=00$ - 70 - 70, F2= $80-70-70$ y F3 $120-70-70$ kg/ ha y D1 $=1.5 \mathrm{~m}$ * $0.25 \mathrm{~m}$ y D2 $=1.5 \mathrm{~m}$ * $0.35 \mathrm{~m}$, obteniéndose los tratamientos: $\mathrm{T} 1=\mathrm{F} 1$ * D1, $\mathrm{T} 2$ $=\mathrm{F} 1{ }^{\star} \mathrm{D} 2, \mathrm{~T} 3=\mathrm{F} 2{ }^{\star} \mathrm{D} 1, \mathrm{~T} 4=\mathrm{F} 2{ }^{\star} \mathrm{D} 2, \mathrm{~T} 5=\mathrm{F} 3{ }^{*} \mathrm{D} 1$ y T6=F3 * D2, estas dosis se aplicaron a los 17 y 62 días después de la siembra. Luego se evaluó desde la siembra hasta la cosecha y los datos se procesaron mediante análisis de varianza y Duncan, también se analizó la concentración de nitrógeno y la densidad estomática por tratamiento. Los resultados determinaron que el T5 sobresalió en longitud de planta con $129.3 \mathrm{~cm}$, rendimiento comercial con 13.25 tn/ha y T4 en peso de vainas por planta con 633.4 g y número de vainas por planta con 51 . En análisis foliar el T6 con $6.69 \%$ de nitrógeno y densidad estomática el T5 con 98 estomas/ $\mathrm{mm} 2$. Se concluye que, a mayor dosis de nitrógeno y menor distanciamiento, que es T5 con 98 estomas $/ \mathrm{mm} 2$ y concentración de $5.83 \mathrm{~g}$ de nitrógeno/100 g de materia seca influyeron en el rendimiento.

Palabras clave: Nitrógeno; Densidad de siembra, Densidad estomática, Arveja; Rendimiento
ABSTRACT

The objective was to determine the stomatal density, by electron microscopy, of pea leaves grown at different doses of nitrogen and distances between plants, to obtain higher yields. Therefore, the experimental area was installed using the Completely Random Block Design, with six treatments consisting of F1 $=00$ - $70-70, F 2=80-70-70$ and F3 $120-70-70 \mathrm{~kg} /$ ha and D1 $=1.5 \mathrm{~m} * 0.25 \mathrm{~m}$ and D2 $=1.5 \mathrm{~m} * 0.35 \mathrm{~m}$, obtaining the treatments: $\mathrm{T} 1=\mathrm{F} 1{ }^{\star} \mathrm{D} 1, \mathrm{~T} 2=\mathrm{F} 1$ * $\mathrm{D} 2, \mathrm{~T} 3=\mathrm{F} 2{ }^{*} \mathrm{D} 1, \mathrm{~T} 4=\mathrm{F} 2{ }^{*} \mathrm{D} 2, \mathrm{~T} 5=\mathrm{F} 3{ }^{*} \mathrm{D} 1$ and $\mathrm{T} 6$ $=F 3{ }^{*} \mathrm{D} 2$, these doses were applied at 17 and 62 days after sowing. Then it was evaluated from sowing to harvest and the data were processed by analysis of variance and Duncan, the nitrogen concentration and stomatal density were also analyzed by treatment. The results determined that T5 excelled in plant length with $129.3 \mathrm{~cm}$, commercial yield with $13.25 \mathrm{tn}$ / ha and T4 in weight of pods per plant with $633.4 \mathrm{~g}$ and number of pods per plant with 51 . In foliar analysis, T6 with $6.69 \%$ of nitrogen and stomatal density in $\mathrm{T} 5$ with 98 stomata / mm2. It is concluded that the higher the nitrogen dose and the lower the spacing, which is T5 with 98 stomata / $\mathrm{mm} 2$ and a concentration of $5.83 \mathrm{~g}$ of nitrogen / $100 \mathrm{~g}$ of dry matter, influenced the yield

Key words: Nitrogen; Planting density Stomatal density; Pea; Yield

\section{RESUMO}

O objetivo foi determinar a densidade estomática, por microscopia eletrônica, de folhas de ervilha cultivadas em diferentes doses de nitrogênio e distâncias entre plantas, para obter maiores rendimentos. Portanto, a área experimental foi instalada utilizando-se o Delineamento em Bloco Completamente Aleatório, com seis tratamentos consistindo de F1 = $00-70-70, F 2=80-70-70$ e F3 120 $70-70 \mathrm{~kg} /$ ha e $\mathrm{D} 1=1,5 \mathrm{~m} * 0,25 \mathrm{me} \mathrm{D} 2=1,5$ $m$ * $0,35 m$, obtendo os tratamentos: $\mathrm{T} 1=\mathrm{F} 1$ * $\mathrm{D} 1, \mathrm{~T} 2=\mathrm{F} 1$ * $\mathrm{D} 2, \mathrm{~T} 3=\mathrm{F} 2$ * D1, T4 = F2 * D2, $\mathrm{T} 5=\mathrm{F} 3{ }^{*} \mathrm{D} 1$ e $\mathrm{T} 6=\mathrm{F} 3{ }^{\star} \mathrm{D} 2$, essas doses foram aplicadas aos 17 e 62 dias após a semeadura. Em seguida, foi avaliada da semeadura à colheita e os dados foram processados por análise de variância e Duncan, a concentração de nitrogênio e a densidade estomática também foram analisadas por tratamento. Os resultados determinaram que $\mathrm{T} 5$ se destacou em comprimento de planta com $129,3 \mathrm{~cm}$, rendimento comercial com 13,25 th / ha e T4 em peso de vagens por planta com 633,4 ge número de vagens por planta com $51 . \mathrm{Na}$ análise foliar, T6 com 6,69\% de nitrogênio e densidade estomática em T5 com 98 estômatos / mm2. Conclui-sé que quanto maior a dose de nitrogênio e menor o espaçamento, que é T5 com 98 estômatos / $\mathrm{mm} 2$ e concentração de 5,83 g de nitrogênio / $100 \mathrm{~g}$ de matéria seca, influenciaram no rendimento.

Palavras-chave: Nitrogênio; Densidade de plantio Densidade estomática; Ervilha e rendimento 


\section{INTRODUCCIÓN}

Durante muchos años, los agricultores han aplicado diversos fertilizantes sintéticos a base de nitrógeno $(\mathrm{N})$ de cantidad variada sin tener medidas de dosificación referenciales; esto se debe por el desconocimiento de la concentración de este nutriente en el suelo y el distanciamiento de siembra, los cuales han influido en el desarrollo de la planta y por ende en el rendimiento y calidad de grano del cultivo de arveja. Puesto que mencionar que el aumento de la utilización de $\mathrm{N}$ se debe esencialmente al aumento de la capacidad de absorción de $\mathrm{N}$ y de la capacidad de transferencia de N. Por lo tanto, deben considerarse densidades de siembra más altas $\mathrm{y}$ tasas de aplicación de $\mathrm{N}$ reducidas para promover una mejor utilización de $\mathrm{N}$ y rendimiento de grano con menores costos ambientales en la producción (1). Para lo cual, se requiere un mayor uso de fertilizantes inorgánicos para hacer frente a la creciente de manda de alimentos.

Asimismo, es importante mencionar que el nitrógeno del suelo utilizado es limitado; ya sea por sus procesos químicos como mineralización, nitrificación, desnitrificación, inmovilización, lixiviación y volatilización, hace que se pierda, lo que reduce la disponibilidad de nutrición para el cultivo de arveja. Esto se debe a que el suelo, con mayor contenido de materia orgánica, presentó un mayor potencial de desnitrificación, un mayor número más probable de desnitrificantes y emitió más $\mathrm{N}_{2} \mathrm{O}$ a la madurez del cultivo que el suelo. También, la productividad y dinámica de los ecosistemas terrestres está limitada a la disponibilidad de nutrientes (2). También, para las plantas la disponibilidad de nitrógeno $(\mathrm{N})$ es la principal limitante en la productividad de los cultivos, que junto con el fósforo $(P)$ determinan el crecimiento vegetal (3).

Debido a la deficiencia del nitrógeno en el suelo, es importante aplicar este nutriente como fertilizante sintético en el cultivo de arveja; puesto que influye de manera favorable para incrementar el rendimiento. Sin embargo, la productividad parcial del factor es baja, en comparación a la referencia internacional $y$, una eficiencia agronómica adecuada en productividad, por el uso del nitrógeno proveniente de los fertilizantes sintéticos (4).

También es necesario mencionar que el uso continuo de estos fertilizantes ha influido en la contaminación ambiental en el suelo y agua. Puesto que las formas móviles de $\mathrm{N}$ en sí mismas tienen consecuencias medioambientales. Aunque la mayoría de los aportes de nitrógeno satisfacen necesidades humanas como la producción agrícola, sus consecuencias medioambientales son graves y duraderas (5). Por lo que también, se puede utilizar como alternativas fertilizantes solidos o líquidos derivados de la industria alimentaria como por ejemplo efluentes tratados después de la refinación de aceite y con variantes en el procesamiento para promover nutrientes en concentración importante, entre ellos el Nitrógeno, resultando una alternativa orgánica y sostenible.

Es necesario resaltar, que ha menor distanciamiento de siembra, se incrementa 
la competencia nutricional entre plantas y disminuye la disponibilidad del nitrógeno en el suelo, resultando deficiente; sin embargo, se incrementa el rendimiento, pero se reduce la calidad de grano de arveja. Este análisis se puede relacionar con el incremento de la densidad de plantas que promovió reducción significativa en el número de legumbres, granos y granos por legumbre por planta, contrario al aumento observado en el peso de campo por parcela (g) y rendimiento $(\mathrm{kg} / \mathrm{ha})(6)$.

Por este motivo, se realiza la investigación para determinar que distanciamiento de siembra y que dosis de nitrógeno es adecuado para obtener mayor rendimiento y calidad de grano en el cultivo de arveja.

\section{MATERIALES Y MÉTODOS}

El experimento se realizó en el distrito de Barrio Tres Piedras, Supe Puerto, provincia de Barranca ubicado en el departamento de Lima con las coordenadas de Latitud Sur $10^{\circ} 48^{\prime}$ 16.468" y Longitud Oeste 77 44' 34.496" altitud de 20 m.s.n.m. la temperatura es de $19.3^{\circ} \mathrm{C}$.

\section{Factor de estudio}

El fertilizante de nitrógeno (Urea) se aplicó a los 17 y 62 días después de la siembra al cultivo de arveja, para lo cual se tuvo en cuenta las dosis y distanciamiento que emplean los agricultores de la zona y el análisis de fertilización de suelo, lo cual permitió establecer los tratamientos. Es necesario mencionar que el rango de estimaciones experimentales de la proporción y cantidad de $\mathrm{N}_{2}$ fijado por importantes leguminosas como arveja es de 17 a $244 \mathrm{Kg} / \mathrm{ha}$ (7). Además de tener en cuenta la competencia nutricional entre planta; para lo cual es importante el distanciamiento adecuado para el mayor rendimiento. Estos componentes de rendimiento correspondientes a número de vainas por planta, número de granos por vaina, rendimiento en vaina verde, rendimiento en grano seco y peso de 100 semillas, que afectan negativamente por la mayor densidad de siembra de arveja (666666 plantas por hectárea) (8).

\section{Fertilización}

Para establecer la fertilización de nitrógeno se tuvo en cuenta las dosis que emplean los agricultores de la zona y el análisis de suelo, obteniéndose de esta manera la formulación de macro nutrientes que se detalla en la Tabla 1.

Tabla 1. Dosis de fertilización de nitrógeno.

\begin{tabular}{cccc}
\hline Fertilización & \multicolumn{2}{c}{ Dosis de nitrógeno (kg./ha) } \\
\hline$F_{1}$ & 00 & $\mathbf{P}$ & $\mathbf{K}$ \\
$\mathrm{F}_{2}$ & 80 & 80 & 80 \\
$\mathrm{~F}_{3}$ & 120 & 80 & 80 \\
\hline
\end{tabular}




\section{Distanciamientos}

En las medidas de entre planta y entre surco que se detalla en la Tabla 2, se tomaron en cuenta en base a las mediciones que usualmente emplean los agricultores de la zona.

Tabla 2. Distanciamientos de siembra entre planta y surco del cultivo de arveja.

\begin{tabular}{ccc}
\hline Densidad & Medidas de siembra* & Plantas por hectárea \\
\hline $\mathrm{D}_{1}$ & $1.6 \mathrm{~m} .{ }^{*} 0.25 \mathrm{~m}$. & 50000 \\
$\mathrm{D}_{2}$ & $1.6 \mathrm{~m} .{ }^{*} 0.30 \mathrm{~m}$. & 41666 \\
\hline
\end{tabular}

Distanciamiento entre surco * distanciamiento entre planta.

\section{Tratamiento}

Para establecer los tratamientos se tuvo en cuenta las combinaciones ordenadas de fertilización y distanciamiento. Obteniéndose de esta manera las interacciones que se detalla en la Tabla 3.

Tabla 3. Fertilización y densidad de siembra de arveja.

\begin{tabular}{cc}
\hline Tratamientos & Interacción \\
\hline$T_{1}$ & $F_{1} \times D_{1}$ \\
$T_{2}$ & $F_{1} \times D_{2}$ \\
$T_{3}$ & $F_{2} \times D_{1}$ \\
$T_{4}$ & $F_{2} \times D_{2}$ \\
$T_{5}$ & $F_{3} \times D_{1}$ \\
$T_{6}$ & $F_{3} \times D_{2}$ \\
\hline
\end{tabular}

\section{Procedimientos}

Se llevó muestra de suelo al Instituto Nacional de Innovación Agraria (INIA) - Huaral, para determinar la concentración de nitrógeno del suelo (Tabla 3).

Para la instalación se empleó el modelo estadístico del Diseño de Bloques Completamente al Azar, que constó de 6 tratamientos y 3 bloques.
Seguido se aplicaron los siguientes fertilizantes industriales: como fuente de nitrógeno $\mathrm{N}_{2} \mathrm{O}$, como fuente de fósforo $\mathrm{P}_{2} \mathrm{O}_{5} \mathrm{y}$ como fuente de potasio $\mathrm{K}_{2} \mathrm{O}$.

Luego se fertilizó a los 17 y 62 días después de la siembra al cultivo de arveja, como consta en la tabla 3 
Continuamente las evaluaciones se realizaron desde la siembra hasta la cosecha y los datos obtenidos se procesaron con análisis de varianza.

Luego se tomaron muestras representativas de hojas de cada tratamiento y se llevaron INIA - Huaral, para determinar la concentración de nitrógeno en cada distanciamiento por último se hizo el mismo procedimiento con el análisis biológico, para determinar la densidad estomática.

\section{Análisis estadístico}

Obtenidos los datos de las evaluaciones, se procesaron con el análisis de varianza con arreglo factorial $2 \star 3$ (3 dosis de fertilización de nitrógeno y 2 distanciamientos), lo cual se comparó los datos con la distribución de Fisher al $5 \%$ de error. Estos procedimientos se realizaron en los parámetros de evaluación; lo que permitió determinar si hubo efecto en alguno de los tratamientos. Seguido se aplicó la prueba múltiple de Duncan que permitió conocer que tratamiento destaca con relación a los demás. En la tabla 4 se detalla los componentes del análisis de varianza de dos factores.

Tabla 4. Análisis de varianza para el diseño factorial $a \times b$.

\begin{tabular}{llllll}
\hline FV & SC & GL & CM & $\mathbf{F}_{0}$ & Valor-p \\
\hline Efecto $A$ & $\mathrm{SC}_{\mathrm{A}}$ & $\mathrm{a}-1$ & $\mathrm{CM}_{\mathrm{A}}$ & $\mathrm{CM}_{\mathrm{A}} / C M_{\mathrm{E}}$ & $\mathrm{P}\left(\mathrm{F}>\mathrm{F}_{0}^{\mathrm{A}}\right)$ \\
Efecto $B$ & $\mathrm{SC}_{\mathrm{B}}$ & $\mathrm{b}-1$ & $\mathrm{CM}_{\mathrm{B}}$ & $\mathrm{CM}_{\mathrm{B}} / \mathrm{CM}_{\mathrm{E}}$ & $\mathrm{P}\left(\mathrm{F}>\mathrm{F}_{0}^{\mathrm{B}}\right)$ \\
Efecto $\mathrm{AB}$ & $\mathrm{SC}_{\mathrm{AB}}$ & $(\mathrm{a}-1)(\mathrm{b}-1)$ & $\mathrm{CM}_{\mathrm{AB}}$ & $\mathrm{CM}_{\mathrm{AB}} / \mathrm{CM}_{\mathrm{E}}$ & $\mathrm{P}\left(\mathrm{F}>\mathrm{F}_{0}^{\mathrm{AB}}\right)$ \\
Error & $\mathrm{SC}_{\mathrm{E}}$ & $\mathrm{ab}(\mathrm{n}-1)$ & $\mathrm{CM}_{\mathrm{E}}$ & & \\
\hline Total & $\mathrm{SC}_{\mathrm{T}}$ & $\mathrm{abn}-1$ & & & \\
\hline
\end{tabular}

Fuente: “Análisis y diseño de experimentos" (9)

RESULTADOS Y DISCUSIÓN

De acuerdo al análisis de suelo realizado en el INIA - Huaral se aprecia en la Tabla 5, que las concentraciones de macronutrientes son bajo de nitrógeno $(0.1 \%-0.2 \%)$, potasio (125ppm-250 ppm) y fósforo (12ppm-36ppm). También presenta baja concentración de materia orgánica (2\% - 4\%), no presenta peligro de sales, su pH es neutro (6.5 -75); sin embargo, es recomendable aplicar compuestos como compost $u$ otros fertilizantes que tengan compuestos de macro y micronutrientes. Cabe mencionar que el tipo de suelo es franco arenoso. 
Tabla 5. Análisis de suelo del cultivo de arveja.

\begin{tabular}{|c|c|c|c|c|c|c|c|c|c|c|c|c|}
\hline \multirow{2}{*}{ Sector } & \multirow{2}{*}{$\begin{array}{l}\text { C.E. } \\
\mathrm{ms} / \mathrm{cm}\end{array}$} & \multirow{2}{*}{ pH } & \multirow{2}{*}{ M.O. \% } & \multirow{2}{*}{$\mathbf{N} \%$} & \multirow{2}{*}{$\underset{\text { ppm }}{\text { P }}$} & \multirow{2}{*}{$\begin{array}{c}\mathrm{K} \\
\mathrm{ppm}\end{array}$} & \multirow{2}{*}{$\begin{array}{c}\mathrm{CaCO} 3 \\
\%\end{array}$} & \multicolumn{4}{|c|}{$\begin{array}{c}\text { Cationes intercambiables } \\
\text { Meq / } 100 \mathrm{~g} \text { suelo }\end{array}$} & \multirow{2}{*}{$\mathrm{CICl}-\mathrm{E}$} \\
\hline & & & & & & & & $\mathrm{Ca}$ & Mg & $\mathrm{Na}$ & K & \\
\hline Tres Piedras & 1.50 & 7.13 & 1.31 & 0.075 & 8 & 98 & 0.88 & 14.06 & 0.87 & 0.19 & 0.24 & 15.36 \\
\hline
\end{tabular}

Fuente: “Análisis Básico de Fertilidad” Número de Laboratorio 211. (10).

Respecto a la de fertilización para el cultivo de arveja, INIA -Huaral determinó las medidas de macro nutrientes recomendable de N, P y K, lo cual permitió establecer las dosis de los tratamientos (Ver Tabla 6).

Tabla 6. Recomendación de fertilización para el cultivo de arveja.

\begin{tabular}{rrrr}
\hline Cultivo & Dosis (kg/ha) & & \\
\hline \multirow{2}{*}{ Arveja } & $\mathrm{N}$ & $\mathrm{P}$ & $\mathrm{K}$ \\
& 80 & 80 & 80 \\
\hline
\end{tabular}

Fuente: “Análisis Básico de Fertilidad” Número de Laboratorio 211. (10).

\section{Evaluaciones de las características físicas}

En cuanto a los resultados de las características física que se muestra en la Tabla 7, se determinó que el T5 obtuvo mayor rendimiento con $13.252 \mathrm{tn} / \mathrm{ha}$, lo cual se interpreta que a menor distanciamiento y mayor dosis de nitrógeno se obtuvo mayor rendimiento.

Tabla 7. Características físicas de acuerdo a los tratamientos.

\begin{tabular}{|c|c|c|c|c|c|c|c|}
\hline Tratamientos & $\begin{array}{l}\text { Longitud de } \\
\text { planta }(\mathrm{cm})\end{array}$ & Tratamientos & $\begin{array}{l}\text { Peso de vainas } \\
\text { por planta (g) }\end{array}$ & Tratamientos & $\begin{array}{l}\text { Número de } \\
\text { vainas por } \\
\text { planta }\left(\mathbf{N}^{\circ}\right)\end{array}$ & Tratamientos & $\begin{array}{c}\text { Rendimiento } \\
\text { comercial } \\
\text { (tn/ha) }\end{array}$ \\
\hline$T_{5}$ & $129.303 \mathrm{a}$ & $\mathrm{T}_{4}$ & $633.4 \mathrm{a}$ & $\mathrm{T}_{4}$ & 50.911 a & $T_{5}$ & $13.252 \mathrm{a}$ \\
\hline $\mathrm{T}_{4}$ & $124.103 \mathrm{ab}$ & $T_{5}$ & $545.6 \mathrm{a}$ & $T_{6}$ & $47.222 \mathrm{a}$ & $T_{6}$ & $12.156 \mathrm{ab}$ \\
\hline$T_{6}$ & $123.503 \mathrm{ab}$ & $\mathrm{T}_{2}$ & $450.9 \mathrm{a}$ & $\mathrm{T}_{2}$ & $46.978 \mathrm{a}$ & $\mathrm{T}_{4}$ & $11.752 \mathrm{ab}$ \\
\hline$T_{2}$ & $121.263 \mathrm{~b}$ & $T_{3}$ & $426.6 \mathrm{a}$ & $T_{5}$ & $43.955 \mathrm{a}$ & $\mathrm{T}_{2}$ & 11.231ab \\
\hline
\end{tabular}




\begin{tabular}{|c|c|c|c|c|c|c|c|}
\hline Tratamientos & $\begin{array}{l}\text { Longitud de } \\
\text { planta }(\mathrm{cm})\end{array}$ & Tratamientos & $\begin{array}{l}\text { Peso de vainas } \\
\text { por planta (g) }\end{array}$ & Tratamientos & $\begin{array}{l}\text { Número de } \\
\text { vainas por } \\
\text { planta }\left(\mathbf{N}^{\circ}\right)\end{array}$ & Tratamientos & $\begin{array}{c}\text { Rendimiento } \\
\text { comercial } \\
\text { (tn/ha) }\end{array}$ \\
\hline $\mathrm{T}_{3}$ & $119.525 \mathrm{~b}$ & $\mathrm{~T}_{6}$ & $403.3 \mathrm{a}$ & $\mathrm{T}_{3}$ & $40.978 \mathrm{a}$ & $\mathrm{T}_{3}$ & $9.623 \mathrm{~b}$ \\
\hline $\mathrm{T}_{1}$ & $118.587 \mathrm{~b}$ & $\mathrm{~T}_{1}$ & $348.6 \mathrm{a}$ & $\mathrm{T}_{3}$ & $37.733 \mathrm{a}$ & $\mathrm{T}_{3}$ & $8.963 b$ \\
\hline C. variación & $2.80 \%$ & & 33.68 & & 15.36 & & 14.99 \\
\hline $\begin{array}{l}\text { Significancia } \\
(5 \%) F^{\star} D\end{array}$ & Significativo & & $\begin{array}{l}\text { No } \\
\text { Significativo }\end{array}$ & & $\begin{array}{l}\text { No } \\
\text { Significativo }\end{array}$ & & $\begin{array}{l}\text { No } \\
\text { Significativo }\end{array}$ \\
\hline
\end{tabular}

Nota: Letras iguales son estadísticamente homogéneos.

\section{Análisis foliar de nitrógeno}

Concerniente al análisis foliar analizado en INIA - Huaral que se detalla en la tabla 8 , se determinó que, a mayor distanciamiento y mayor dosis de nitrógeno, que es $\mathrm{T}_{6}$ obtuvo $6.69 \mathrm{~g}$ de nitrógeno/100 $\mathrm{g}$ de materia seca; sin embargo, que a menor distanciamiento con mayor dosis de nitrógeno que es $\mathrm{T}_{5}$ con $5.83 \mathrm{~g}$ de nitrógeno/ $100 \mathrm{~g}$ de materia seca sobresalió en rendimiento y se encuentra dentro de la calificación normal. Lo cual se interpreta que a una adecuada concentración de nitrógeno influyó en el rendimiento

Tabla 8. Análisis foliar de nitrógeno en hojas de arveja por tratamiento.

\begin{tabular}{cccccc}
\hline Tratamientos & Interacción & Resultados (\%) & Calificación & Valores normales & $\begin{array}{c}\text { Rendimiento } \\
\text { (tn/ha) }\end{array}$ \\
\hline $\mathrm{T}_{1}$ & $\mathrm{~F}_{1} \times \mathrm{D}_{1}$ & 3.48 & Bajo & $4.50-6.00$ & 8.963 \\
$\mathrm{~T}_{2}$ & $\mathrm{~F}_{1} \times \mathrm{D}_{2}$ & 3.02 & Bajo & $4.50-6.00$ & 11.231 \\
$\mathrm{~T}_{3}$ & $\mathrm{~F}_{2} \times \mathrm{D}_{1}$ & 5.36 & Normal & $4.50-6.00$ & 9.623 \\
$\mathrm{~T}_{4}$ & $\mathrm{~F}_{2} \times \mathrm{D}_{2}$ & 5.62 & Normal & $4.50-6.00$ & 11.752 \\
$\mathrm{~T}_{5}$ & $\mathrm{~F}_{3} \times \mathrm{D}_{1}$ & 5.83 & Normal & $4.50-6.00$ & 13.252 \\
$\mathrm{~T}_{6}$ & $\mathrm{~F}_{3} \times \mathrm{D}_{2}$ & 6.69 & Alto & $4.50-6.00$ & 12.156 \\
\hline
\end{tabular}

Fuente: "Análisis de porcentaje de nitrógeno en hojas" Número de Laboratorio $(11,12)$.

\section{Nitrógeno utilizado por tratamiento}

Respecto al del consumo de nitrógeno total por tratamiento que se muestra en la Tabla 9 , que, mediante métodos de peso de hectárea de suelo, factor de Van Bemmelen, Factor de conversión de nitrógeno total a disponible a ppm en relación a Carbono a nitrógeno $(\mathrm{C} / \mathrm{N})$ y conversiones, se determinó el mayor consumo de nitrógeno en el $\mathrm{T}_{5} \mathrm{y}_{6}$; sin embargo, el $\mathrm{T}_{5}$ con $1565.75 \mathrm{Kg} / \mathrm{ha}$ de consumo total de nitrógeno destacó en rendimiento comercial con 13.25 tn/ha. 
Tabla 9. Total, de nitrógeno utilizado (kg.), de acuerdo a las dosis de aplicación.

\begin{tabular}{cccccc}
\hline Tratamientos & Interacción & $\begin{array}{c}\text { Dosis de } \\
\text { nitrógeno } \mathbf{( k g / h a}\end{array}$ & $\begin{array}{c}\text { Nitrógeno } \\
\text { en el suelo } \\
\mathbf{( k g / h a )}\end{array}$ & $\begin{array}{c}\text { Total, de } \\
\text { nitrógeno } \\
\text { utilizado } \\
\mathbf{( k g / h a )}\end{array}$ & $\begin{array}{c}\text { Rendimiento } \\
\text { (tn/ha) }\end{array}$ \\
\hline $\mathrm{T}_{1}$ & $\mathrm{~F}_{1} \times \mathrm{D}_{1}$ & 0 & 36.75 & 36.75 & 8.963 \\
$\mathrm{~T}_{2}$ & $\mathrm{~F}_{1} \times \mathrm{D}_{2}$ & 0 & 36.75 & 36.75 & 11.231 \\
$\mathrm{~T}_{3}$ & $\mathrm{~F}_{2} \times \mathrm{D}_{1}$ & 80 & 36.75 & 116.75 & 9.623 \\
$\mathrm{~T}_{4}$ & $\mathrm{~F}_{2} \times \mathrm{D}_{2}$ & 80 & 36.75 & 116.75 & 11.752 \\
$\mathrm{~T}_{5}$ & $\mathrm{~F}_{3} \times \mathrm{D}_{1}$ & 120 & 36.75 & 156.75 & 13.252 \\
$\mathrm{~T}_{6}$ & $\mathrm{~F}_{3} \times \mathrm{D}_{2}$ & 120 & 36.75 & 156.75 & 12.156 \\
\hline
\end{tabular}

Fuente: "Análisis de porcentaje de nitrógeno en hojas" Número de Laboratorio $(11,12)$.

\section{Densidad estomática por tratamiento}

Con el uso del microscopio electrónico de Barrido para el grado de aumento de $800 \mathrm{X}$, se obtuvo la micrografía por tratamiento, lo cual permitió determinar que la mayor densidad de estomas se obtuvo el $\mathrm{T}_{6}$ con 241.93 estomas/ $\mathrm{mm}^{2}$, Por lo que se analiza que a medida que se incrementa la concentración de nitrógeno y se aumenta en distanciamiento incrementó la densidad de estomas; sin embargo el $\mathrm{T}_{5}$ con 98.79 estomas $/ \mathrm{mm}^{2}$ sobresalió en rendimiento, por lo que a medida que se incrementó el nitrógeno y disminuyó en distanciamiento obtuvo mayor rendimiento (ver Tabla 10).

Tabla 10. Número de estomas por mm2 por tratamiento.

\begin{tabular}{lcc}
\hline Tratamientos & Interacción & Densidad estomática (Estomas/mm2) \\
\hline $\mathrm{T} 1(00 / 70 / 70) 1,5 \times 0,25$ & $\mathrm{~F}_{1} \times \mathrm{D}_{1}$ & 221.77 \\
$\mathrm{~T} 2(00 / 70 / 70) 1,5 \times 0,35$ & $\mathrm{~F}_{1} \times \mathrm{D}_{2}$ & 40.32 \\
$\mathrm{~T} 3(80 / 70 / 70) 1,5 \times 0,25$ & $\mathrm{~F}_{2} \times \mathrm{D}_{1}$ & 207.66 \\
$\mathrm{~T} 4(80 / 70 / 70) 1,5 \times 0,35$ & $\mathrm{~F}_{2} \times \mathrm{D}_{2}$ & 193.55 \\
$\mathrm{~T} 5(120 / 70 / 70) 1,5 \times 0,25$ & $\mathrm{~F}_{3} \times \mathrm{D}_{1}$ & 98.79 \\
$\mathrm{~T} 6(120 / 70 / 70) 1,5 \times 0,35$ & $\mathrm{~F}_{3} \times \mathrm{D}_{2}$ & 241.93 \\
\hline
\end{tabular}




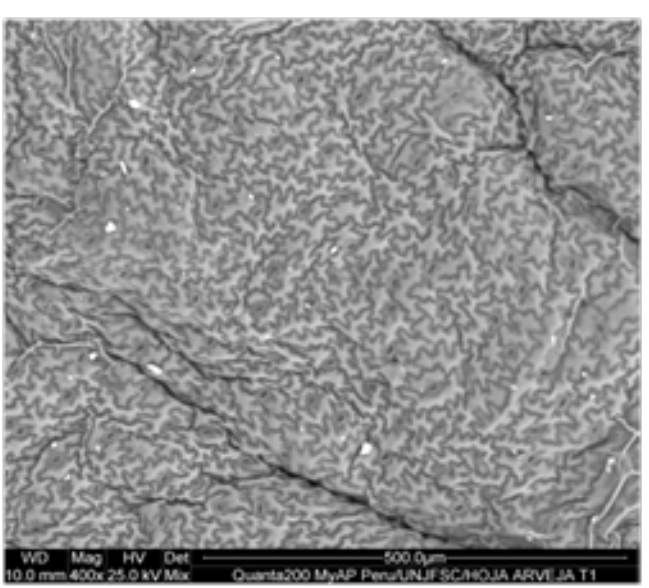

$\mathrm{T}_{1}$

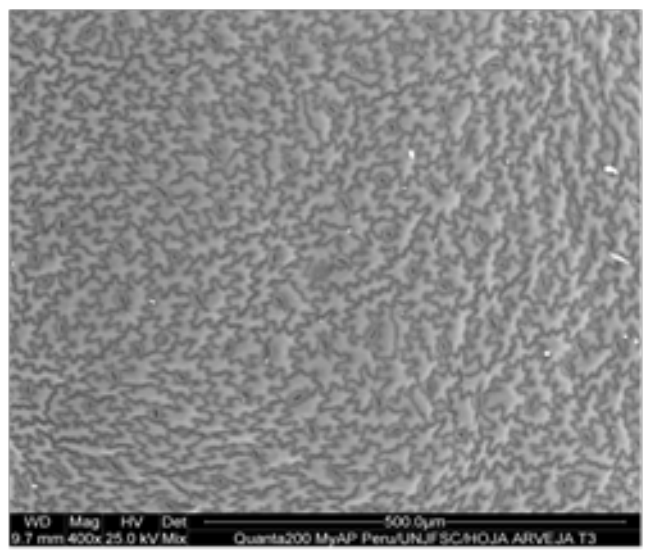

$\mathrm{T}_{3}$

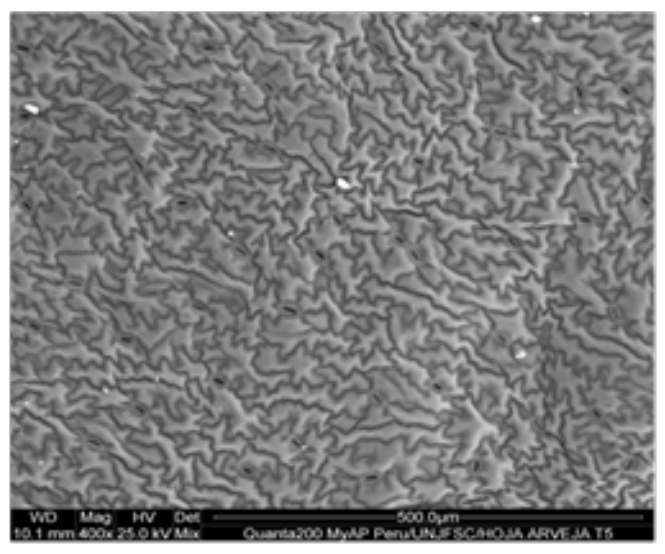

$\mathrm{T}_{5}$

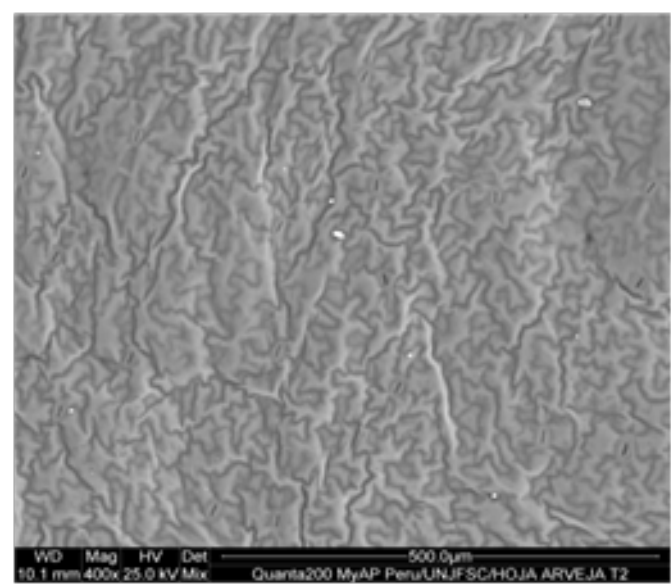

$\mathrm{T}_{2}$

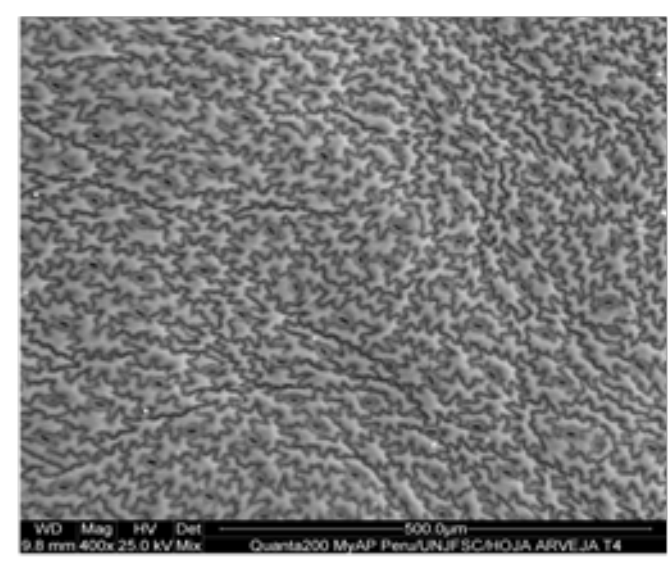

$\mathrm{T}_{4}$

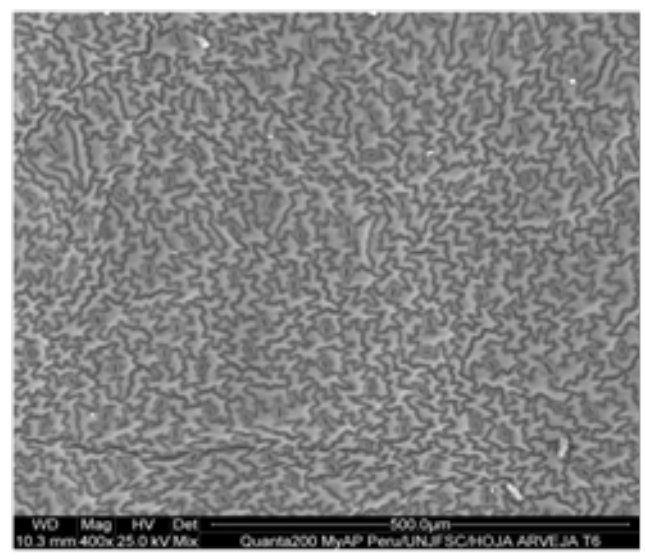

$T_{6}$

Figura 1. Micrografía de estomas de cada tratamiento. 


\section{Discusión}

\section{Evaluaciones de las características físicas}

De acuerdo a los resultados de las características físicas del cultivo de arveja que se detalla en la tabla 7 , se indica que no hubo significancia estadística de las variables de rendimiento; es decir la aplicación de nitrógeno no influyeron en el rendimiento. Sin embargo, a una adecuada dosis que se aprecia en el tratamiento $\mathrm{T}_{5}$ con $13.25 \mathrm{tn} /$ ha sobresalió con relación a los demás tratamientos. Por lo que la utilización de $\mathrm{N}$ se debe esencialmente al aumento de la capacidad de absorción de $\mathrm{N}$ y de la capacidad de transferencia de N. Por lo tanto, deben considerarse densidades de siembra más altas y tasas de aplicación de $\mathrm{N}$ reducidas para promover una mejor utilización de $\mathrm{N}$ y rendimiento de grano (1).

\section{Análisis foliar de nitrógeno}

Obtenidos los resultados de la concentración de nitrógeno en hojas por tratamiento que se indica en la tabla 8 , se destaca que el $\mathrm{T}_{5}$ con $5.83 \mathrm{~g}$ de nitrógeno/ $100 \mathrm{~g}$ de materia seca obtuvo mayor rendimiento. Por lo tanto, a esta concentración de nitrógeno fue óptimas para muchas reacciones bioquímicas en la formación de aminoácidos, proteínas y otros compuestos. Pues este elemento es esencial para el crecimiento vegetal, es el elemento más frecuentemente limitante en los suelos agrícolas. El nitrógeno se requiere en cantidades más altas que cualquier otro elemento (además del carbono, hidrógeno y oxígeno), ya que es un elemento importante de muchos componentes bioquímicos incluyendo proteínas, aminoácidos y ácidos nucleicos (12).

\section{Nitrógeno utilizado por tratamiento}

El consumo de nitrógeno total utilizado por tratamiento que se muestra en la tabla 9, se obtuvo mediante métodos de peso de hectárea de suelo, factor de Van Bemmelen, Factor de conversión de nitrógeno total a disponible a ppm en relación a Carbono a nitrógeno $(\mathrm{C} / \mathrm{N})$ y conversiones. Se determinó que el tratamiento $T_{5}$ con $156.75 \mathrm{Kg}$ de nitrógeno total $\mathrm{Kg} / \mathrm{ha}$ obtuvo el mayor rendimiento con 13.25 Tn/ha. Por lo tanto, a esta cantidad de total de nitrógeno utilizado por hectárea, la disponibilidad de este nutriente fue favorable para el aprovechamiento en muchas reacciones bioquímicas de aminoácidos, proteínas, formación de carbohidratos y otras reacciones. Pues este cultivo de arveja tiene la capacidad de fijación de nitrógeno y por lo general es muy alta. Se han medido aportes de hasta $185 \mathrm{~kg} /$ ha por esta vía (13) (14).

\section{Densidad estomática por tratamiento}

En cuanto a la densidad estomática, que mediante evaluaciones de microscopía electrónica de barrido en hojas representativa por tratamiento que se indica en la tabla 10, se obtuvo que el $\mathrm{T}_{6}$ con 241.93 estomas/ $\mathrm{mm}^{2}$, que es a mayor dosis de nitrógeno y distanciamiento, sobresalió con relación a los demás; sin embargo, el T5 con 98 estomas $/ \mathrm{mm}^{2}$, que es a mayor dosis de nitrógeno y menor distanciamiento destacó en rendimiento. Por lo tanto, el T5 con 98 
estomas $/ \mathrm{mm}^{2}$ es el indicador de densidad de estomas que obtuvo mayor rendimiento. Es importante resaltar que estas características de las estomas y su concentración pueden ser factores determinantes en las diferencias en producción de biomasa entre genotipos (15).

\section{CONCLUSIONES}

Se determinó que la densidad estomática T5 con 98 estomas/mm2 obtuvo mayor rendimiento comercial con 13.252 tn/ha; por lo tanto, en este tratamiento se aprovechó de manera óptima la aplicación de nitrógeno y en menor distanciamiento, resultando el indicador adecuado de densidad estomática.

También se determinó que en la densidad estomática T5, se obtuvo la calificación normal de la concentración de nitrógeno en hojas; lo que se evidencia que las mayores aplicaciones del nitrógeno no influyeron en sus concentraciones. Por lo tanto, estas características biológicas y químicas influyeron en el rendimiento

\section{REFERENCIAS BIBLIOGRÁFICAS}

1. Xu C, Huang S, Tian B, Ren J, Meng Q y Wang P. Manipulación de la densidad de siembra y la aplicación de fertilizantes nitrogenados para mejorar el rendimiento y reducir el impacto ambiental en la producción de maíz chino. Rev. Frontiers in Plant Science, [revista en internet], 2017;8:1234, [acceso 3 de octubre de 2021]. Disponible en DOI: [https://doi.org/10.3389/ fpls.2017.01234]

2. Illarze G, Del Pino A, Riccetto $S$ y Irisarri P. Emisión de óxido nitroso, nitrificación, desnitrificación y mineralización de nitrógeno durante el cultivo del arroz en 2 suelos de Uruguay. Revista Argentina de Microbiología [revista en internet], 2018;50(1):97-54, [acceso 5 de octubre de 2021]. Disponible en DOI: [https://doi.org/10.1016/j.ram.2017.05.004]

3. Cerón Rincón, LE y Aristizábal Gutiérrez FA. Dinámica del ciclo del nitrógeno y fósforo en suelos. Revista Colombiana de Biotecnología [revista en internet]. Colombia, 2012; 14(1):285295. [acceso 1 de octubre de 2021]. Disponible en [https://www.redalyc.org/articulo. oa?id=77624081026]

4. Ríos MJ, Gómez Martínez J., Bolaños Aguilar RE y Gutiérrez Matamoros CJ. Fertilización sintética y orgánica y su efecto en la producción de maíz, variedad Nutrinta Amarillo. Revista la calera [revista en internet], 2019; 19 (32):4147 [acceso 2 de octubre de 2021]. Disponible en: DOI: [https://doi.org/10.5377/calera. v19i32.8439]

5. Vitousek PM, Aber CJ, Howarth RW, Likens GE, Matson PA, Schindler DW, Schlesinger WH y Tilman GD. Alteración Antrópogenica del Ciclo Global del Nitrógeno: Causas y Consecuencias. [internet]. Tópicos en ecología, publicado por la sociedad norteamericana de ecología; 1997 [acceso 30 de setiembre de 2021] N ${ }^{\circ} 1$. Disponible en https://www.esa.org/ wp-content/uploads/2013/03/numero1.pdf

6. Garcés Fiallos FR y Vera Alcívar AM. Enfermedades y componentes de rendimiento en líneas de fréjol bajo tres densidades de siembra. Revista Agronomía Mesoamericana, [revista en internet]. 2014; 25(1): 169-180. [acceso 28 de setiembre 2021]. Disponible en [http://www.mag.go.cr/rev_meso/v25n01_169. pdf]

7. Peoples MB, Herridge DF y Ladha JK. Fijación biológica de nitrógeno: ¿Una fuente eficiente de nitrógeno para la producción agrícola sostenible? [Biological nitrogen fixation: An efficient source of nitrogen for sustainable agricultural production?] Plant and Soil, [revista en internet]. 1995; 174: 3-28. [acceso 25 de setiembre 2021]. Disponible en [http:// www.lavierebelle.org/IMG/pdf/biological_ nitrogen_fixation_-_an_efficient_source_ 
of_nitrogen_for_sustainable_agricultural_ production.pdf]

8. Casanova L, Solarte J, y Checa O. Evaluación de cuatro densidades de siembra en siete líneas promisorias de arveja arbustiva (Pisum sativum L.). Revista de Ciencias Agrícolas, [revista en internet] 2012; 29(2): 129-140. [acceso 23 de setiembre 2021] disponible en [https://dialnet. unirioja.es/servlet/articulo?codigo=5104145]

9. Gutiérrez $H$, De La Vara R, Cano A, y Osorio M. Análisis y diseño de experimentos. [libro en Internet] Libro, 2da Edición. Centro Universitario de Ciencias Exactas e Ingeniería y Centro de Investigación de Matemáticas. México, 2008. [acceso 25 de setiembre de 2021]. Disponible en [https://gc.scalahed.com/ recursos/files/r161r/w19537w/analisis_y_ diseno_experimentos.pdf]

10. Instituto Nacional de Innovación Agraria (INIA) - Huaral. Análisis básico de fertilidad. Hoja análisis de suelo del sector Chacarita Supe Puerto. Laboratorio de Suelos de Instituto Nacional de Innovación Agraria - Huaral. Perú. 2018

11. Instituto Nacional de Innovación Agraria (INIA) - Huaral. Análisis de porcentaje de nitrógeno en hojas. Hoja análisis de foliar en el cultivo de arveja. Laboratorio de Suelos de Instituto Nacional de Innovación Agraria Huaral, 2018.

12. Barrera Cobos OA, y Sáenz Vélez M. El aprovechamiento del nitrógeno por la planta con tecnología $\mathrm{N}-\mathrm{HIB} \circledast$. Revista Ingenio, [revista en internet], 2016; 12(1): 85-99. [acceso 1 de octubre de 2021]. Disponible en https:// revistas.ufps.edu.co/index.php/ingenio/ article/view/2127

13. Rennie RJ, y Dubetz $S$. Nitrogen-15Determined Nitrogen Fixation in Field-Grown Chickpea, Lentil, Fababean, and Field Pea. Agronomy journal [revista en internet], 1986; 78:654-660 [acceso 2 de octubre de 2021]. Disponible en [https://pubag.nal.usda.gov/ catalog/1474406]

14. Prieto GM. Pautas para el manejo del cultivo de Arveja. Agencian de Estación Rural Arroyo Seco - Instituto Nacional de Tecnología Agropecuaria. [revista en internet], 2010; [acceso 26 de setiembre de 2021]. Disponible en: [https://inta.gob.ar/sites/default/files/ script-tmp-pautas-para-el-manejo-del-cultivode-arveja-final.pdf]

15. Álvarez-Holguín A, Morales-Nieto CR, Corrales-Lerma R, Avendaño-Arrazate $\mathrm{CH}$, Rubio-Arias HO, y Villarreal-Guerrero $\mathrm{F}$. Caracterización estomática, concentración de clorofila y su relación con producción de biomasa en Bouteloua curtipendula. Agronomía Mesoamericana, [revista en internet] 2018; 29(2), 251-261. Disponible en: DOI: [https://doi.org/10.15517/ma.v29i2.29900] 